\title{
Evaluation of bond strength in lithium disilicate ceramics subjected to different times and methods of removal of hydrofluoric acid residues
}

\begin{abstract}
Objective: To evaluate the bond strength in lithium disilicate ceramics subjected to different times and methods of removal of conditioning residues.

Materials and methods: Bonding strength was evaluated in lithium disilicate discs after conditioning with hydrofluoric acid, the samples were subjected to different times and methods of removal: air-water spray, ultrasonic bath with distilled water $2 \mathrm{~min}$, ultrasonic bath with distilled water $4 \mathrm{~min}$, phosphoric acid $2 \mathrm{~min}$, phosphoric acid $4 \mathrm{~min}$. Cylinders were made of flowable composite resin that were positioned on the discs. The samples were subjected to micro-shear tests on a universal testing machine.
\end{abstract}

Results: The two-way ANOVA test and the Pos Hoc Tukey test $(\mathrm{p}<0.05)$ revealed that the ultrasonic bath group with distilled water 2 min presented significant difference with respect to the phosphoric acid $4 \mathrm{~min}$; however, it did not present significant difference with respect to the other groups.

Conclusion: The ultrasonic bath removal method for 2 min presented the highest value of bonding strength.

Keywords: Ceramics, Hydrofluoric Acid, Microshear bond strength
Volume 12 Issue 5 - 202 I

\author{
Raquel Yokebed Guerra Pérez,' Anthony \\ Pierre Bolaños Trujillo,' Bruna Fortes \\ Bittencourt, ${ }^{4}$ Pablo Chavez Alayo, ${ }^{1,2}$ Diego \\ Alonso Melendez Murillo,' John Alexis \\ Dominguez ${ }^{3}$ \\ 'Especialidad de Rehabilitación Oral Universidad Perruana \\ Cayetano Heredia, Facultad de Estomatologia, Peru \\ ${ }^{2}$ Maestria Universidad Perruana Cayetano Heredia, Facultad de \\ Estomatologia, Peru \\ ${ }^{3}$ Maestria y Doctorado, Professor Universidad Peruana \\ Cayetano Heredia, Facultad de Estomatologia, Peru \\ ${ }^{4}$ Universida Estatal de Ponta Grossa, Dentitry, Brasil
}

\begin{abstract}
Correspondence: John Alexis Dominguez, Maestria y
Doctorado, Professor Universidad Peruana Cayetano Heredia,

Facultad de Estomatologia, Av. Gral. Salaverry 2475, San Isidro

15076, Lima Peru, Tel +5 | 949725704, Fax +5 |319000 I,

Email johnalexis.dominguez@gmail.com
\end{abstract}

Received: March 22, 202I | Published: November 19, 202

\section{Introduction}

Worldwide, ceramics have increased their demand, this is because in addition to providing high aesthetics, also have an adequate resistance. ${ }^{1}$ Therefore, the professional must have knowledge of the adhesive protocol which varies according to the type of ceramic to work with. Gracis et al. $^{2}$ proposed a new classification system of ceramics in which they are categorized into three families: glass-matrix ceramics, polycrystalline ceramics and resin-matrix ceramics. Among the glass-matrix ceramics, the lithium disilicate (IPS e.max ${ }^{\circledR}$ Ivoclar Vivadent-Schaan Liechtenstein), which consists of aluminosilicate glass to which lithium oxide is added..$^{2,3}$

Glass-matrix ceramics are sensitive to the conditioning technique, which means the adhesion of resin cement materials is improved by creating micro-retentive surfaces through hydrofluoric acid (HF) conditioning. ${ }^{4}$ This method of conditioning generates micromorphological changes in the surface area of glass-matrix synthetic ceramics in which the hydrofluoric acid reacts with the silica-containing glass matrix to form hexafluorsilicates, a selective subtraction of the glass matrix and exposing the crystalline phase. ${ }^{4,5}$ The HF conditioning develops a significant amount of precipitate from the residual by-products, salts that are insoluble and crystalline detritus (chemical compound of the residuals) that remain coupled to the surface of the ceramic called smear vitrius, obstructing the microporosities and preventing the penetration of the adhesive system and the resin. ${ }^{4-7}$ The removal of the residues that occur the application of $\mathrm{HF}$ is a very important step within the bonding protocol since by avoiding it, we can diminish the adhesion and therefore obtain failures in the treatments.

Giraldo ${ }^{8}$ evaluated the effect of passive and active application of $37 \%$ phosphoric acid for $30 \mathrm{~s}$ as a method of removing residues produced after HF conditioning on the bond strength of lithium disilicate glass matrix synthetic ceramics. It was concluded that the active application with $37 \%$ phosphoric acid increases the bond strength values between the resin cement and the lithium disilicate ceramic.

Bruzi et al. ${ }^{9}$ in their study conclude that the removal method with ultrasonic bath with distilled water and the application of silane is important to improve the bond strength. Ortega et al. ${ }^{10}$ evaluated the effect of different methods of post-conditioning removal of glassmatrix ceramics such as lithium disilicate and micro-shear bond strength values comparing five methods: control group 1 (air-water spray), group 2 (active application of phosphoric acid 37\% for 10 seconds), group 3 (ultrasonic bath with alcohol for 5 minutes) and group 4 (active application with alcohol for 10 seconds). The values obtained were not statistically different from the control group, but no study evaluates longer cleaning time with the mentioned methods. Recongnizing two possible post-conditioning cleaning techniques with hydrofluoric acid and not having a recommended time or longer evaluations.

Therefore, the aim of this study was to evaluate the bonding strength of lithium disilicate glass matrix ceramics subjected to different times and methods of removal of hydrofluoric acid residues. 
We hypothesize that the longer the post-conditioning time presents better values of resistance of union to micro-shear in lithium disilicate.

\section{Material and methods}

After authorization from the ethics committee of the city of Lima with number (CAREG-ORVEI-060-19), 25 lithium disilicate glass matrix synthetic ceramic discs were made (IPS emax Press, Ivoclar - Vivadent; Schaan, Liechtenstein) according to the studies of the area, low-translucency (LT) ingot, shade A2, $12 \mathrm{~mm}$ in diameter and $2 \mathrm{~mm}$ in height. Were made in a wax pattern and pressed according to the manufacturer's recommendations, all discs were subjected to the firing protocol after injection into an oven. (Programat EP 3010 Ivoclar Vivadent, Dental Ceramic Furnace).

The samples were polished with truncated-cone diamond burs (838L - Jota, Switzerland) on both sides to obtain the standardized samples.

The lithium disilicate (LD) glass matrix synthetic ceramic discs (IPS emax Press, Ivoclar - Vivadent; Schaan, Liechtenstein) (Table I) were inserted into a PVC tube (Matusita, Tigre S.A.; Lima, Peru) with the following dimensions: $20 \mathrm{~mm}$ high and $19 \mathrm{~mm}$ in diameter. They were fixed with transparent self-curing acrylic Vitacryl (Vitalloy, Lima, Peru), to ensure that the disc does not suffer any movement as a result of the polymerization of the acrylic, they were fixed with double contact adhesive tape (TopEx; Lima, Peru) to a glass plate. Once the acrylic was polymerized, the surface of the ceramic disc was made uniform with sandpaper with the following microns: 400, 600 , 800 (Asalite, P400A-5305; Lima, Peru) of $10 \times 10 \mathrm{~cm}$, using abundant water, before and after sanding procedure for 10 times with an interval of 1 minute (Sanding water - only water).

\section{Conditioning of the ceramics}

The LD were etched with 9\% hydrofluoric acid (Porcelain Etch; Ultradent, Utah, USA) for $20 \mathrm{~s}$, then the material was removed according to the study group:

Five groups were formed with different methods and removal times

Control: Air spray and filtered water for $1 \mathrm{~min}$.

BU- 2M: Ultrasonic bath immersed in distilled water for $2 \mathrm{~min}$ (Table 2).

BU - 4M: Ultrasonic bath immersed in distilled water for 4 min (Table 2).

AF - 2M: 37\% phosphoric acid (Table 1) was applied for $2 \mathrm{~min}$ activated with a microbrush (Table 2).

AF - 4M: 37\% phosphoric acid (Table 1) was applied for $4 \mathrm{~min}$ activated with of a microbrush (Table 2).

The groups are described in the Table 2.

Table I Materials used in the study with their respective manufacturers

\begin{tabular}{ll}
\hline Material - manufacturer & Brand name \\
\hline Lithium Disilicate (Ivoclar - Vivadent) & IPS emax Press \\
$9 \%$ hydrofluoric acid (Ultradent) & Porcelain Etch \\
$37 \%$ phosphoric acid (Ultradent) & Ultra Etch \\
Silane (Ultradent) & Silane \\
Adhesive (3M ESPE) & Adper Single Bond 2 \\
Flowable resin (3M ESPE) & Filtek Flow Z350 \\
\hline
\end{tabular}

Table 2 Study Group

\begin{tabular}{lllll}
\hline & Ultrasonic & $\begin{array}{l}\text { Phosphoric } \\
\text { acid }\end{array}$ & $\begin{array}{l}\mathbf{2} \\
\text { minutes }\end{array}$ & $\begin{array}{l}\mathbf{4} \\
\text { minutes }\end{array}$ \\
\hline $\begin{array}{l}\text { CONTROL } \\
(n=5)\end{array}$ & & & & \\
$\operatorname{BU}-2 M(n=5)$ & $X$ & & $X$ & \\
$\operatorname{BU}-4 M(n=5)$ & $X$ & & $X$ \\
$\operatorname{AF}-2 M(n=5)$ & & $X$ & $X$ & \\
AF-4M $(n=5)$ & & $X$ & & $X$ \\
\hline
\end{tabular}

$X$, procedure performed in the group

\section{Washing and drying the discs}

All the discs after having been subjected to the removal method corresponding to their group were subjected to spray washing with filtered water and air for 1 min and drying with air spray for $20 \mathrm{~s}$.

\section{Application of silane and adhesive system on glass matrix ceramics}

Silane (Silane; Ultradent, Utah, USA) was applied with the help of a microbrush on the surface of the disilicate glass matrix ceramic disk, allowing it to rest for $60 \mathrm{~s}$ and with the help of a triple syringe air was applied for $10 \mathrm{~s}$. Then, the adhesive was applied with the help of a microbrush making a constant rubbing on the surface applying a layer, it was polymerized for $20 \mathrm{~s}$ with a $1470 \mathrm{~mW} /\left[\mathrm{cm} \rrbracket^{\wedge} 2 \mathrm{LED}\right.$ light curing unit $\left(\llbracket \llbracket 3 \mathrm{M} \rrbracket^{\wedge} \mathrm{TM}\right.$ Elipar $\rrbracket^{\wedge} \mathrm{TM}$ DeepCure-L;St.Paul,NM,USA ) at a distance of $2 \mathrm{~mm}$ and with a power of $1470 \mathrm{~mW} /\left[\mathrm{cm} \rrbracket^{\wedge} 2\right.$ according to the manufacturer's instructions, checking that the charge is always complete.

\section{Placement of the tygon}

Flowable resin) were made with the help of a tygon (Aigocath BD: Cundinamarca, Colombia) $2 \mathrm{~mm}$ high by $0.8 \mathrm{~mm}$ in diameter which were positioned on the surface of the ceramic disks, making a constant pressure for the fixation with the help of tweezers and photocuring for 40 seconds with a $1470 \mathrm{~mW} /\left[\mathrm{cm} \rrbracket^{\wedge} 2\right.$ LED light curing unit After photocuring the resin cylinder, the silicone coating of the tygon was cut off with the help of a new $\mathrm{N}^{\circ} 11$ scalpel blade for each tygon used, removing the tygon with the remains of flowable resin, obtaining the flowable resin cylinders on the lithium disilicate glass matrix synthetic ceramic. The resin cylinders were made one by one, so that the already made cylinders were not over-exposed to the light of the light curing unit.

Eight flowable resin cylinders were placed per disc, checking that there is a $2 \mathrm{~mm}$ distance between the cylinders, placed at random, adding up to a total of 120 flowable resin cylinders.

\section{Micro-shear}

The ceramic discs were subjected to micro-shear tests after being stored for 24 hours in saline solution at room temperature after the resin cylinders had been cemented. The tests were carried out in a semi-universal testing machine (0M 100 - Odeme Dental Research; Brazil) with the help of an orthodontic wire $\mathrm{N}^{\circ} 7$ bent in 8 (Morelli Ortodontia; Brazil), with a load cell of $50 \mathrm{~N}$, at a speed of 0.75 $\mathrm{mm} / \mathrm{m}$, until the moment of failure. One hundred and twenty sets of bent wires were used to use one wire per resin cylinder. The tests were performed at the dental materials laboratory of the Universidad Peruana Cayetano Heredia - Salaverry. All tests were performed by the researcher in one day, after training by an expert. 


\section{Failure analysis}

Fractured specimens were observed qualitatively under optical microscopy at $40 \times$ magnification. The failure modes were classified as follows: adhesive (ceramic-resin cement interface), cohesive (in the resin cement) and mixed (involving adhesive and cohesive failures). A descriptive analysis was performed, obtaining the averages and standard deviation of the resistance according to the groups analyzed. The normality of the data was evaluated with the D'Agostino test. Bivariate analysis was performed with the two-way ANOVA test and the Post Hoc Tukey test. The present study used a 95\% confidence level $(\mathrm{p}<0.05)$. The statistical program Bioestat 5.0 was used.

\section{Results}

In vitro comparison of two removal methods was carried out, which were phosphoric acid and ultrasonic bath with distilled water for 2 and 4 minutes, post surface conditioning with hydrofluoric acid in lithium disilicate ceramics. In addition, a control group was set up, which was only cleaned with air and water spray.

When evaluating the results of the 2-minute phosphoric acid group, it showed the following micro-shear bond strength value in megapascals (Mpa) (AF - 2M: $4.84 \pm 1.75 \mathrm{Mpa}$ ) which was higher compared to the AF- 4M group: $3.62 \pm 1.12 \mathrm{Mpa}$. with statistically significant difference $(\mathrm{P}<0.05)$ (Table 3$)$.

The AF-4M group presented the lowest value of micro shear bonding resistance $(3.62 \pm 1.12 \mathrm{Mpa})$; presenting a statistically significant difference $(\mathrm{P}<0.05)$. with respect to the other groups (Table 2) and control group (Figure 1).

The results with the other groups can be observed in Table 3. The failure analysis is expressed in Table 4.

Table 3 Mean and standard deviation of micro-shear bond strength values in megapascals $(\mathrm{MPa})$ of different post-conditioning removal techniques with hydrofluoric acid in lithium disilicate glass matrix synthetic ceramics at different times

\begin{tabular}{lll}
\hline Time & AF & BU \\
\hline $2 \mathrm{M}$ & $4.84 \pm 1.75 \mathrm{aA} *$ & $7.29 \pm 2.4 \mathrm{IaA} \alpha$ \\
$4 \mathrm{M}$ & $3.62 \pm 1.12 \mathrm{bB} \mathrm{A}^{*}$ & $5.83 \pm 2.49 \mathrm{aA} \alpha$ \\
Control & $6.31 \pm 2.49 \mathrm{a}$ &
\end{tabular}

AF, Phosphoric acid; BU, Ultrasonic bath; 2M, 2 minutes; 4M, 4 minutes

Different small letters denote significant difference between experimental and control groups

Different capital letters denote significant vertical difference between working times

$\alpha$ *, Different symbols denote significant difference horizontally

Table 4 Failure mode (\%) for the different surface treatments and adhesive systems

\begin{tabular}{lllll}
\hline & & ADHESIVE & COHESIVE & MIXED \\
\hline $2 M$ & AF & 85 & 12 & 3 \\
& $\mathrm{BU}$ & 95 & 5 & 0 \\
$4 \mathrm{M}$ & $\mathrm{AF}$ & 80 & $1 \mathrm{I}$ & 9 \\
& $\mathrm{BU}$ & 83 & $1 \mathrm{I}$ & 6 \\
CONTROL & & 91 & 9 & 0 \\
\hline
\end{tabular}

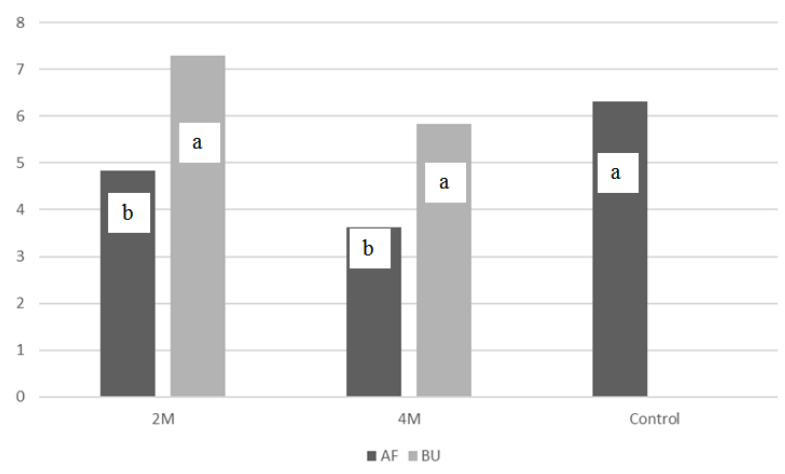

Figure I Mean and standard deviation of micro-shear bond strength values in megapascals $(\mathrm{MPa})$ of different post-conditioning removal techniques with hydrofluoric acid in lithium disilicate glass matrix synthetic ceramics at different times.

AF, Phosphoric acid; BU, Ultrasonic bath; 2M, 2 minutes; 4M, 4 minutes.

Different small letters denote significant difference between experimental and control groups.

\section{Discussion}

This work evaluated two techniques as a method of removing the residues produced by the use of $9 \% \mathrm{HF}$ as a conditioning agent in lithium disilicate glass matrix synthetic ceramics. Tests were performed by separating into five groups: application of $37 \%$ phosphoric acid for 2 and 4 minutes and an ultrasonic bath with distilled water for 2 and 4 minutes, and a control group where air and water spray was applied. The method of removal of the residuals with $37 \%$ phosphoric acid for 4 minutes (AF-4M) presented the lowest value compared to the other groups presenting a significant difference.

It is important to mention that surface treatments with hydrofluoric acid at $9 \%$ and the removal of post-conditioning residues are used for better bond strength values, such treatments are necessary to modify the ceramic surface because they usually present low surface energy which prevents the penetration of bonding agents. ${ }^{13,14}$ In the present study, the samples that were subjected to the application of $37 \%$ phosphoric acid as a removal method obtained significantly lower bond strength values than the samples subjected to ultrasonic baths. Steinhauser ${ }^{12}$ valuated different methods of removal after conditioning with hydrofluoric acid at $10 \%$ of feldspathic glass matrix synthetic ceramics, the techniques evaluated were air and water spray for 1 minute, ultrasonic bath with distilled water for 5 minutes, $37 \%$ phosphoric acid for 1 minute and $37 \%$ phosphoric acid for 1 minute and ultrasonic bath for 5 minutes, finding no significant differences between them; however, our procedures were carried out in lithium disilicate ceramics, which belong to the same group according to Gracis, ${ }^{2}$ obtaining results without significant difference, but, an increase in bond strength values is observed when ultrasound is applied for two minutes.

On the other hand, in our study, the samples of the group with $37 \%$ phosphoric acid for 4 minutes presented the lowest bond strength values $(3.62 \pm 1.12 \mathrm{MPa})$. This can be attributed to the fact that $37 \%$ phosphoric acid not only serves as a method to remove $9 \%$ hydrofluoric acid, but also generates more sodium, potassium and calcium residues that form a layer between the glass matrix synthetic ceramic and the resin cement, which decreases the bond strength. ${ }^{4}$

In order to eliminate the residues that obliterate the surface of glassmatrix synthetic ceramics, several authors mention the ultrasonic bath removal method with distilled water as the most effective, however, 
there is no study that mentions or compares the times for an effective result. ${ }^{16,17}$ Marques $^{17}$ report that they evaluated different methods of post-conditioning removal with hydrofluoric acid in feldspathic glass matrix synthetic ceramics, which were water and air spray for 4 minutes, ultrasonic bath with distilled water for 4 minutes, ultrasonic bath with acetone for 4 minutes and ultrasonic bath with alcohol for 4 minutes. As a result, the ultrasonic bath with distilled water for 4 minutes showed higher bonding strength values compared to the other groups Bruzi ${ }^{9}$ mentioned that when evaluating different methods of post-conditioning removal with $5 \%$ hydrofluoric acid, they concluded that the method with ultrasonic bath with distilled water for 2 minutes presented the highest value of bond strength. When comparing the values obtained in the present study with respect to the ultrasonic bath group with distilled water for 2 min with the study of Bruzi ${ }^{9}$ we can highlight that the values were higher than those obtained in our study, using the same time and method of removal of the hydrofluoric acid residues. These superior results may be due to three points: 1 . In the study by Bruzi et al. after standardizing the surface of the ceramics with sandpaper, the samples were immersed in an ultrasonic bath with distilled water for 5 minutes, which could help the surface to be free of contaminants. 2. The diameter of the tygon was 2.4 millimeters $(\mathrm{mm})$ compared to 0.8 millimeters $(\mathrm{mm})$ in our study, ${ }^{18}$ and finally the protocols usedin the two studies.

The low values of microshear bond strengthin this study, indicate the probable variability of this methodology, ${ }^{19}$ In our study, the varibles evaluated such as excessive time subjected to ultrasound and the application of phosphoric acid, could influence the low values. With respect to the BU method of removal, when comparing bond strength values for 2 and 4 minutes, it is observed that the longer the exposure time, the lower the bond strength values. so it rejects the hypothesis raised in this study.

\section{Conclusion}

Under the conditions of this study and its limitations we can conclude:

The method of ultrasonic bath removal with distilled water for 2 minutes presented the highest value of bond strength. The longer the application time of phosphoric acid, the lower the bond strength values.

\section{Funding statement}

The authors did not obtain any funding, the research is part of the training of specialists at the Universidad Peruana Cayetano Heredia.

\section{References}

1. Gildo S, Coelho Santos MJ, Rizkalla A. Adhesive cementation of etchable ceramic esthetic restorations. J Can Dent Assoc. 2009;75(5):379-384.

2. Gracis S, Thompson VP, Ferencz JL. A new classification System for AllCeramic and Ceramic - like Restorative Materials. Int J Prosthodont. 2015;28(2):227-235.
3. Shenoy A, Shenoy N. Dental Ceramics:An Update. J Conser Dent 2010;13(4).

4. Belli R, Guimarães JC, Filho AM, et al. Post-etching cleaning and resin/ ceramic bonding:microtensile bond strength and EDX analysis. J Adhes Dent. 2010;12:295-303.

5. Zogheib LV, Bona AD, Kimpara ETet al. Effect of hydrofluoric acid etching duration on the roughness and flexural strength of a lithium disilicate - based glass ceramic. Braz Dent J. 2011;22(1):45-50.

6. Canay S, Hersek N, Ertan A. Effect of different acid treatments on a porcelain surface. J Oral Rehabil 2001;28:95-101.

7. Leite F, Ozcan M, Valandro L, et al. Effect of the Etching Duration and Ultrasonic Cleaning on Microtensile Bond Strength Between Feldspathic Ceramic and Resin Cement. The Journal of Adhesion. 2013;89(3): 159-173.

8. Giraldo T, Villada V, Castillo M, et al. Active and Passive Aplication of the phosporic acid on the Bond strength of lithium disilicate. Brazilian Dental Journal. 2016;27(1):90-94.

9. Bruzi G, Carvalho A, Giannini M, et al. Post-etching cleaning influences the resin shear bond strength to CAD/CAM lithium disilicate ceramics. Appl Adhes Sci. 2017;5(17).

10. Ortega LF, Reis AF, Rodrigues JA, et al. Effect of different postetching cleaning protocols on microshear bond strength of a lithium disilicate-reinforced ceramic, and SEM evaluation. $J$ Clin Dent Res.2016;13(2):38-48.

11. Al-Jeaidi ZA. Influence of Post- Etch Crystalline Residue on the Bond Strength of Lithium Disilicate Ceramic An in vitro Study. J Pak Dent Assoc. 2016; 25(2):74-77.

12. Steinhauser H, Tursff C, Franca F, et al. Microshear bond strength and surface micromorphology of a feldspathic ceramic treated with different cleaning methods after hydrofluoric acid etching. J Appl Oral Sci. 2014;22(2):85-90.

13. Ho G. Insights on Ceramics as Dental Materials. Part II:Chemical Surface Treatments. Silicon (2011) 3:117-123.

14. Jardel V, Degrange M, Picard B, et al. Surface energy of etched ceramic. Int J Prosthodont. 1999;12(5):415-418.

15. Peumans M, Van Meerbeek B, Yoshida Y, et al. Porcelain veneers bonded to tooth structure:an ultra-morphological FE-SEM examination of the adhesive interface. Dent Mater. 1999;15(2):105-119.

16. Magne P, Cascione D. Influence of post-etching cleaning and connecting porcelain on the microtensile bond strength of composite resin to feldspathic porcelain. J Prosthet Dent. 2006;96:354-361.

17. Marquez M, Pereira F, Cavalcanti J. Does the ultrasonic cleaning medium affect the adhesion of resin cement to feldespathic ceramic? J Adhes Dent. 2012;14:507-509.

18. Sirisha K, Rambabu T, Shankar YR, et al. Validity of bond strength tests:A critical review:Part I. J Conserv Dent. 2014;17(4):305-311.

19. Sirisha K, Rambabu T, Ravishankar Y, et al. Validity of bond strength tests:A critical review-Part II. J Conserv Dent. 2014;17(5):420-426. 